\title{
Procédés interactifs de construction du sens dans le discours. \\ Un modèle dans le développement des compétences orales au niveau avancé en langue étrangère ?
}

\author{
Interactive procedures in meaning construction as models \\ in developing complex foreign language oral competences
}

\author{
Bernadeta Wojciechowska \\ Université Adam Mickiewicz de Poznań \\ bewoj@amu.edu.pl \\ Weronika Wilczyńska \\ Université Adam Mickiewicz de Poznań \\ wil@amu.edu.pl
}

\begin{abstract}
This article is aimed at giving a part of response when it comes to selecting resources, both relevant and easily accessible, which could serve as models in developing complex L2 oral competences. Those resources should encompass all levels (relational, semantic, and formal) required in realizing and interpreting efficiently our communicative intentions in oral language. Among suggested criteria, priority is clearly given to the mastery of interactive procedures in meaning construction, as playing a crucial role both in a speaker's/learner's communicative efficacy and the dynamics of his/her oral capacities' development through the media. Moreover, to illustrate our reflection, we analyze the pedagogical and interactive potential in some excerpts from Le téléphone sonne, a radio segment available in podcast format.
\end{abstract}

Keywords: French foreign language didactics, media discourse, oral competences, negotiation of meaning, interaction 


\section{PRÉALABLES}

Pour la didactique du FLE, la question principale face à la problématique du développement des compétences orales consiste à cerner la spécificité de la communication orale et, par conséquent, à définir les capacités des interlocuteurs requises pour y participer en tant qu'acteurs sociaux de plein droit. Il semble que la spécificité en question résulterait non seulement du canal oral par opposition au canal écrit, mais d'une façon plus significative encore du caractère interactif, coconstruit et situé (temps et lieu) du discours oral.

Un coup d'œil rapide sur les descriptifs des niveaux définis par le Cadre Européen Commun de Référence pour les Langues (2001) permet de constater l'importance qui est donnée à partir du niveau B2+ aux sous-compétences discursives et interactives comme constitutives des compétences orales. La conception du Cadre semble corroborée par les recherches en didactique qui mettent le développement des compétences orales en langue étrangère en rapport étroit avec la maîtrise des stratégies interactives de construction du sens (Bartning, 1997 ; Pekarek, 1999) telles que façonnées par le type de discours ${ }^{1}$. En effet, la sensibilité à la manière dont les thèmes sont choisis, négociés et développés interactivement dans les différents domaines d'activité humaine constitue une disposition de l'attention importante pour l'interprétation des intentions des interlocuteurs (en réception) et pour la réalisation de nos propres intentions avec ou les autres (en production). La difficulté à négocier le sens dans les interactions orales, en revanche, non seulement réduirait l'apprenant/interlocuteur sur le plan communicationnel à des échanges appauvris et/ou à une intercompréhension superficielle, mais en plus elle entraverait, sur le plan de l'apprentissage, les transformations du système conceptuel, sémantique et syntaxique s'élaborant dans les conditions d'interaction sociale (p. ex. Bronckart, 1996a et 1996b ; Mondada, Pekarek-Doehler, 2001 ; Górecka, 2006 ; Wojciechowska, 2007). Il en résulte que l'emploi des procédés interactifs de construction du sens, loin d'être une aptitude purement formelle et technique, peut devenir :

1) un outil de communication permettant une participation active et compétente dans la construction de l'intercompréhension et dans la gestion des conversations,

2) un mode d'apprentissage permettant l'accès aux représentations des interlocuteurs étrangers et aux présupposés socio-culturels qui alimentent leurs manières d'agir langagièrement, et par ce biais un moyen d'enrichissement du système conceptuel de l'apprenant par celui véhiculé par les pratiques discursives porteuses de sens dans la culture de la langue étrangère.

\footnotetext{
${ }^{1}$ Nous utilisons le terme discours dans le sens des pratiques communicatives propres à un domaine d'activité sociale.
} 
L'exploitation autonome du potentiel interactif du discours aux fins d'apprentissage de l'oral par l'apprenant nécessite un accès systématique aux représentations sociales actuelles (informations et opinions dominantes) circulant dans l'espace public de la communauté de la langue cible. Cela suppose donc que l'apprenant soit en mesure de choisir dans la multitude des sources d'informations (notamment les médias) celles qui sont valables. Pour accéder aux normes sociales qui régissent l'interaction, il serait essentiel également que l'apprenant puisse les appréhender dans le respect des genres de discours. Plus exactement, il faudrait qu'il envisage le genre comme source d'instructions quant au choix des contenus et des modalités de construction du sens avec l'autre. Et enfin, il faudrait qu'il soit en mesure d'analyser et d'interpréter convenablement les informations et les opinions auxquelles il a accès (tant dans les conversations quotidiennes que dans les médias) afin de les exploiter de manière critique et autonome dans ses propres activités langagières.

Dans le milieu d'enseignement/apprentissage institutionnel de la langue étrangère loin du (ou des) pays de la langue enseignée, l'accès aux ressources peut se faire par l'intermédiaire du podcasting des émissions de radio. Les podcasts de radio, en raison de leur accessibilité (gratuité, possibilité d'écouter à tout moment et à plusieurs reprises, de modifier les conditions d'écoute, par exemple en ralentissant le débit) et surtout en raison de leur qualité informative (actualité, expertise) et discursive (authenticité en termes de pratiques socio-culturelles), pourraient à plus d'un égard constituer des supports didactiques valables (Górecka, 2008). En effet, la radio fait partie des instances qui dans une large mesure reflètent et créent les représentations du monde et agissent sur l'opinion publique. Elle le fait entre autres en donnant la parole dans le cadre d'émissions jouissant d'un certain prestige ${ }^{2}-$ comme $^{2}$ c'est certainement le cas de l'émission Le téléphone sonne diffusée sur France Inter et soumise à notre analyse dans la suite de l'article - à des interlocuteurs ayant des compétences de communication et d'interaction éprouvées et pouvant à ce titre constituer une bonne référence pour les apprenants en langues. On pourrait donc avancer que pour se tenir au courant des événements socio-politiques, ainsi que de l'évolution des mœurs et des idées, reflétée dans les pratiques discursives, un apprenant avancé qui vit loin du pays de la L2 gagnerait à intégrer son développement des capacités d'interaction à l'oral à ses compétences médiatiques ${ }^{3}$.

\footnotetext{
${ }^{2}$ Le prestige de l'émission se reflète entre autres dans le choix des sujets de discussion (demandant une certaine culture générale et parfois même des connaissances spécialisées), dans le choix du registre (souvent soutenu, jamais familier) et aussi dans la qualité des invités (experts du domaine, souvent habiles orateurs).

${ }^{3}$ A ce sujet, il est aussi opportun de remarquer que la compétence orale comprise ainsi répond aux enjeux de l'éducation à la citoyenneté, mise en valeur dans les récents documents du Conseil de l'Europe et du Parlement européen (Recommandation du Parlement Européen et du Conseil du 18 décembre 2006 sur les compétences clés pour l'éducation et la formation tout au long de la vie (2006/962/CE), la capacité à analyser, interpréter et négocier interactivement le sens y constituant une condition sine qua non
} 
Les considérations qui suivent ont un caractère préliminaire. L'objectif à terme de notre travail est justement de contribuer à donner plus de consistance didactique à l'exploitation des podcasts au niveau avancé avec des objectifs qui dépassent leur dimension linguistique ou lexicale. Nous illustrerons ci-après notre projet d'intégrer le développement des compétences médiatiques à celui des compétences orales ${ }^{4}$ justement avec quelques extraits de l'émission déjà évoquée - Le téléphone sonne. Plus exactement, il s'agirait de préciser notamment quels paramètres, en termes d'interactivité, devraient posséder les émissions choisies pour servir à des observations de différente nature, telle vitrine exhibant connaissances et opinions.

\section{INTERACTION DANS LE DISCOURS - DÉFINITIONS}

La notion d'interaction donnant lieu à des définitions différentes, nous nous voyons contraintes de préciser son sens, tel qu'investi dans le présent travail.

À un niveau très général, on recourt au qualificatif « interactif » pour désigner la propriété de tous les actes de parole qui de par leur nature sont toujours adressés à quelqu'un. Selon cette acception, même un discours monologal est interactif dans la mesure où son destinateur prend en compte la représentation du (des) récepteur(s) qu'il vise et à qui il adapte ses dires. Emblématique est, à ce sujet, la position de Charaudeau qui distingue l'interactivité, comme inhérente à tout acte de langage, de l'interlocution qui « concerne la situation locutive de communication - prise de parole alternée entre les deux interlocuteurs » (Charaudeau, 2006, p. 9). Comme le remarque Kerbrat-Orecchioni (2005), ces définitions ne sont pas opérationnelles pour l'analyse du discours et nous rejoignons cette position pour dire qu'elles ne le sont pas non plus pour la didactique, notamment du fait de leur caractère trop englobant. Les interactionnistes réservent le qualificatif interactif aux situations de dis-

de participation active dans la société démocratique, et ceci aussi bien sur le plan de la compréhension que sur le plan d'action efficace.

${ }^{4}$ Dans notre conception, les compétences orales sont envisagées sous l'angle de leurs liens très étroits avec le cadre discursif institutionnalisé de production et de réception de la parole. Dans le cas de l'exploitation des médias, ce cadre discursif relève du domaine public de l'activité sociale dont les pratiques communicatives sont régies par les logiques [démocratiques et commerciales (Burger, 2013, Charaudeau, 2006, 2011, Maingueneau, 2014) et les enjeux (informer, divertir et faire de la publicité Burger (2010)] propres. Il se distingue aussi par les rôles (journaliste, animateur, chroniqueur, etc.) et les modalités de recours aux codes sémiotiques, souvent multiples, précisés et organisés à l'intérieur des genres médiatiques etc. Les particularités de la communication médiatiques appellent des compétences spécifiques qui permettraient à l'apprenant de tenir compte du contexte de production lors de l'interprétation du sens. Etant donné que la compétence médiatique ne fait pas souvent l'objet (tel est le cas en Pologne) d'un enseignement scolaire très poussé, dans la plupart des cas nos étudiants auront besoin non de ré-ancrer les capacités orales qu'ils maîtrisent déjà mais de construire et/ou compléter des compétences nouvelles. 
cours oral qui est élaboré conjointement par au moins deux interlocuteurs se trouvant en même temps dans le même lieu. En revanche, Kerbrat-Orecchioni (2005) propose de caractériser d'interactives seulement les situations de communication orale de face-à-face où chacun des interlocuteurs influe activement sur les actions langagières de l'autre, en modifiant par ses réactions le projet de parole initial de l'autre : « La différence est de taille entre l'approche rhétorique, pour laquelle l'autre n'existe que dans la représentation que s'en fait le locuteur, et l'approche interactionniste, selon laquelle l'autre est bien là en chair et en os, infléchissant en permanence le programme discursif du locuteur, et contrant à l'occasion ses prétentions identitaires » (Kerbrat-Orecchioni, 2005, p. 184). Dans cette vision des choses, l'interaction ne se réduit pas à la forme dialogale (à l'interlocution), l'alternance des tours pouvant être pour ainsi dire programmée par le genre de discours et non susceptible de modifications lors de la rencontre (il suffit de penser aux dialogues didactiques préparés par les apprenants et récités en classe). En effet, l'interaction au sens de KerbratOrecchioni consiste en négociation ici et maintenant et en un effort d'ajustement à trois niveaux essentiels :

- niveau organisationnel qui correspond à ce qui par ailleurs constitue l'objet privilégié de l'analyse conversationnelle, à savoir les mécanismes d'ajustement au niveau des tours de parole, de l'organisation et de la structuration des échanges ;

- niveau interpersonnel qui est celui où se manifestent et s'affirment (et/ou s'affrontent) les identités des interlocuteurs, leurs rôles et leurs places, où s'effectuent, pour reprendre la terminologie goffmanienne, les procédés de figuration ;

- niveau du contenu/sens qui concerne la négociation des thèmes, des informations, des intentions (buts et fonctions) et des opinions (sentiments, attitudes et valeurs). Plus exactement, la négociation suppose une prise de position, une réaction verbale et non verbale visant à évaluer, modifier, réinterpréter, approfondir ou désavouer la parole d'autrui afin de préciser le mieux possible l'objet de l'interaction (p. ex. discussion consensuelle) et/ou d'imposer sa vision de la problématique du monde (p. ex. discussion conflictuelle).

Bien évidemment, dans une interaction, les trois niveaux évoqués brièvement cidessus s'entrelacent, plus encore, ils se conditionnent mutuellement. Du point de vue didactique, il est intéressant d'attirer ici l'attention sur le genre discursif, notion qui explique probablement le mieux l'existence des règles, des scénarios intériorisés auxquels les interlocuteurs se réfèrent plus ou moins consciemment au moment de planifier et d'ajuster leurs actions afin de gérer ensemble l'organisation, la relation et le sens. Il y aurait donc lieu de parler de modèles implicites suffisamment flexibles pour favoriser la négociation (surtout dans les genres conversationnels) ou la limiter (dans les genres fortement ritualisés). 
En fait, de manière générale, l'interactivité au niveau interpersonnel et au niveau organisationnel dans les genres médiatiques d'information et d'opinion ${ }^{5}$ pourrait être qualifiée de faible. Les rôles, les places, la politesse y sont souvent hautement conventionnels ${ }^{6}$ et donc peuvent donner lieu à la constitution de règles de comportement relativement concises et univoques. Du fait de leur haute prévisibilité, ces aspects constitutifs des situations de communication ne posent pas de sérieux problèmes d'enseignement/apprentissage. Il en est de même pour la gestion de l'organisation qui relève majoritairement, dans ce type d'émission, de l'animateur, en limitant l'impact des invités (des experts et, encore plus, des auditeurs qui appellent) sur le déroulement de la rencontre. Par ailleurs, c'est grâce à cette rigidité des schémas discursifs relatifs à la gestion du déroulement et des relations que l'effort des participants à l'interaction peut être investi dans l'élaboration conjointe du sens. Il paraît donc naturel et justifié de se tourner, lors de la didactisation des podcasts de radio d'information et d'opinion, vers le niveau du contenu - en fait, c'est là que l'essentiel des mondes représentés est activé et soumis à l'évaluation, voire à une transformation interactive. Nous suivrons cette logique dans la suite de notre propos sans pour autant sous-estimer l'importance des actions relevant des deux autres niveaux pour l'efficacité de la communication.

\section{DEGRÉ D’INTERACTIVITÉ ET ACCÈS AUX REPRÉSENTATIONS SOCIALES}

Lorsqu'on aborde la question de la construction interactive du sens dans le discours et de l'importance de celle-ci pour le processus d'enseignement/apprentissage d'une langue étrangère, on se rend vite compte que le degré de précision dans la sémantisation des contenus et la profondeur de leur élaboration conjointe ne sont pas les mêmes dans toutes les interactions verbales. En effet, tous les types d'interaction ne demandent pas le même degré d'effort dans la co-construction des objets de discours : cet effort sera réduit quand l'objet du discours sera concret et/ou disponible dans l'environnement physique (p. ex. situations d'achat) (Bange, 1992) ou bien quand son actualisation se fera dans le cadre de genres fortement ritualisés, souvent marqués par l'inégalité des places (p. ex. entretien médical). Le degré d'interactivité peut dépendre également des compétences d'analyse et du répertoire des stratégies de co-construction du sens des locuteurs ainsi que de leur engagement dans cette

\footnotetext{
${ }^{5}$ Voir par exemple une analyse de la gestion de la parole et des rôles assumés par les participants des émissions interactives à la radio dans Becqueret $(2002,2004)$.

${ }^{6}$ Une catégorie spécifique constituent les débats présidentiels où les négociations des rôles, des identités et des tours, comme l'ont démontré les analystes du discours politique, peuvent constituer un vrai enjeu du débat Mitterrand - Chirac, Sarkozy - Royal, en reléguant les problématiques soulevées au second plan.
} 
tâche. Ainsi, un faible degré d'engagement se manifeste en surface, selon Vion (1992), par des réponses uniquement réactives telles que : (1) la production de phatiques d'écoute verbaux et non verbaux, (2) les reprises lexicales accompagnées le plus souvent d'intonation exprimant la position face au contenu ou l'encouragement à poursuivre. Il suffit tout de même que les interlocuteurs tendent vers un consensus ou au contraire qu'ils tiennent à obtenir un avantage pour que les procédés interactifs de co-construction du sens plus avancés, intellectuellement et langagièrement plus exigeants, soient mis en œuvre. Parmi les procédés en question, on peut citer à titre d'exemple la verbalisation des intentions, l'explicitation des présupposés, la négociation de la signification des mots et des concepts-clés, l'évaluation des propos d'autrui, ainsi que toutes sortes de demandes de clarification se faisant au moyen de la modalisation, de la reprise, de la reformulation, de l'implicitation, etc.

Pour l'apprenant avancé en langue étrangère, l'observation des représentations (souvent dynamiques, hétérogènes, floues), soumises par les interactants natifs à la validation sociale, peut donner un accès direct, quoique toujours limité et jamais définitif, à l'imaginaire social d'une communauté discursive donnée. Plus encore, la prise de conscience de la manière dont ces représentations (connaissances et opinions) circulent, sont explicitées, reprises, évaluées, remaniées, etc., nous paraît essentielle pour le développement par l'apprenant du contrôle des processus en question. Et enfin, l'exposition aux podcasts de radio avec plusieurs intervenants, experts et auditeurs qui appellent, constitue également, comme nous l'avons déjà signalé, une source de procédés interactifs nécessaires pour faire évoluer les systèmes conceptuels en relation avec la L2 (et sa culture) et pour participer dans les interactions de manière efficace et autonome.

À la lumière de ce qui précède, les discours choisis pour le développement des compétences orales chez des apprenants adultes (y compris étudiants de philologie romane) au niveau avancé devraient répondre aux critères suivants :

- présenter un caractère oral avec une prédilection pour le discours public dialogal, dialogique et interactif,

- aborder une thématique susceptible d'éveiller l'intérêt des étudiants (problématique socio-culturelle et domaines proches de leur spécialité) correspondant à leurs ambitions intellectuelles et leurs possibilités,

- illustrer la qualité intellectuelle et la dimension critique de la réflexion coconstruite, visible dans la manière de formuler le raisonnement, de se positionner dans le débat et de réagir aux interventions des autres locuteurs,

- déployer une richesse de processus de construction conjointe du discours à tous les niveaux de l'interaction et de leur réalisation par un nombre élevé d'interlocuteurs,

- présenter une variation au niveau de la planification : préfabrication du discours et ajustements tout au long de l'échange,

- impliquer une compréhension du discours exigeant la mise en œuvre des capacités d'analyse et de contrôle des procédés discursifs au niveau conceptuel. 


\section{EXEMPLE D'ÉMISSION}

La réflexion théorique nous a permis de dégager les paramètres des discours oraux se prêtant à une exploitation didactique aux fins de perfectionnement à l'oral au niveau avancé. Passons à présent à l'examen de quelques extraits de l'émission Le téléphone sonne choisie pour observer les critères évoqués plus haut à l'œuvre?

Dans l'émission analysée, les modalités de gestion des tours de parole sont surtout contraintes par le format de l'émission. Il en est de même pour ce qui est du niveau relationnel, fortement institutionnalisé (et donc rarement négocié interactivement) avec des procédés de politesse quasiment rituels, relativement redondants et stables d'une émission à l'autre. Puisque l'observation des stratégies de gestion des tours ainsi que des stratégies de politesse ne demande pas de ressources d'attention importantes, le travail d'écoute peut se focaliser sur l'analyse des conduites des locuteurs visant une construction interactive du sens et sur l'élaboration de l'interprétation personnelle.

\subsection{NÉGOCIATION DU SENS - SÉQUENCES D'INTERACTIVITÉ ÉLEVÉE}

L'émission sert à montrer et à confronter les informations et les opinions venant d'horizons différents, ce qui implique tout un travail d'analyse et d'évaluation interactive des propos d'autrui. Les interlocuteurs-experts présentent leur point de vue, mais ils le font en se rapportant aux appels des auditeurs et aux propos d'autres experts dans le studio sans oublier la prise en compte de l'ensemble des auditeurs de l'émission - en fait, leurs principaux destinataires.

Dans l'exemple ci-dessous, extrait de l'émission du mercredi 4 août 2010, intitulée À quoi servent les ambassadeurs ${ }^{8}$, nous pouvons distinguer des passages qui relèvent aussi bien de l'information que de l'opinion. En même temps, on peut observer comment l'information se développe et se complexifie et comment les opinions sont, plus ou moins explicitement, évaluées par différents intervenants.

\footnotetext{
${ }^{7}$ Dans le contexte didactique, en outre des paramètres discursifs et interactifs des enregistrements proposés en classe, il serait aussi opportun de tenir compte des intérêts des apprenants. Pour cette raison, après avoir exposé les enjeux didactiques et les critères de sélection des documents, il nous semble tout à fait possible et utile de demander aux apprenants de proposer leurs choix.

${ }^{8}$ Les invités de l'émission sont les suivants : André Janier, ancien ambassadeur de France au Qatar, au Tchad, au Yémen, en Irak, puis en Côte d'Ivoire et au Libéria en même temps ; François Nicouleau, ancien ambassadeur de France en Iran ; Franck Renaud, journaliste indépendant et auteur de l'ouvrage Les Diplomates: Derrière la façade des ambassades de France aux Éditions Nouveau Monde ; JeanChristophe Rufin, ancien ambassadeur de France au Sénégal et en Gambie, écrivain et membre de l'Académie Française.
} 
Penchons-nous sur un extrait de l'émission développé autour de la collaboration des ambassadeurs des pays de l'Union européenne (de $16 \min 09$ à $21 \min 23)^{9}$ :

1. $\mathrm{T}$ : Bonsoir je voulais vous demander quelle est quelle est et s'il y a une synergie entre les

2. ambassadeurs de différents pays de l'Union européenne quand ils se trouvent dans une place dans

3. dans un état par exemple s'ils se trouvent eee je sais pas e en Amérique ou ils se trouvent en Arabie

4. Saoudite et cetera il y a une collaboration et synergie entre eux et quel est ce type de synergie quel

5. est ce type de collaboration?

6. PB : Merci pour votre question et bien nous la posons à Franck Renaud qui est le journaliste qui a écrit

7. ce livre sur les diplomates, e est-ce qu'ils travaillent ensemble les diplomates européens ?

8. FR : ee écoutez oui moi l'expérience que j'ai je je je vis et je travaille en en Asie du sud-est je sais que

9. dans le pays où je suis qui est le le Vietnam eee les ambassadeurs des pays européens ou les

10. conseillers conseillers culturels par exemple se retrouvent régulièrement pour des réunions sur des

11. sujets techniques et quand par exemple c'est la France ou l'autre pays c'est l'ambassade du pays qui

12. préside l'Union européenne qui accueille ces réunions

13. PB : Monsieur Nicouleau qui avait été en poste à Téhéran cette cette synergie n'est-elle que technique

14. ou elle va au-delà de est-ce qu'il y a une vraie complicité une solidarité même des pays européens ?

15. FN : Oui une complicité moi j'ai vécu à Téhéran dans une situation un peu spéciale parce que à

16. Téhéran pour des raisons historiques il n'y a pas d'ambassade des États-Unis il est vrai que dans

17. beaucoup de pays l'ambassadeur des États-Unis ou encore les délégués de la Commission européenne

18. sont un peu les grands frères mais les ambassadeurs européens étaient un peu livrés à eux-mêmes et

19. on se retrouvait dans une configuration du type XIX siècle où les grandes puissances étaient encore la

20. Grande Bretagne la Russie la France l'Italie et là du coup ça nous a obligés à travailler ensemble et on a

21. eu une coopération véritablement extraordinaire à cette époque dont je garde un très très bon

22. souvenir

23. PB : Merci André Janier vous avez le même point de vue en Afrique et aussi au Proche Orient où vous

24. avez été

25. AJ : Eh bien pas tout à fait au risque de mettre les pieds dans le plat

26. PB : Il faut il faut

27. AJ : Démentir eee mon ancien collègue François Nicouleau moi je trouve qu'il y a un décalage entre

28. la coopération la coordination la solidarité affichées et la manière dont les affaires secrètes se traitent

29. sur le terrain bon quand il s'agit on se réunit très régulièrement les les européens normalement on

30. doit échanger les informations mais vous imaginez qu'on échange pas toutes les informations et on

31. doit promouvoir des projets de e coordination communs ee quand il s'agit de monter un festival de

32. cinéma ça se passe très très bien pour ce qui est des affaires ee des contrats on peut comprendre

33. en sens inverse que ee ça ne se passe pas aussi sur sur le terrain les les ambassadeurs ne se disent pas

34. tout moi je l'ai vécu mais la plupart du temps à mon niveau ee et chaque ambassadeur a tendance à

35. favoriser à encourager les efforts d'une entreprise de sa nationalité au détriment des autres hein

36. PB : Mhm

37. AJ : Aujourd'hui surtout en période de crise la concurrence est exacerbée

38. PB : On vous perd un peu sur la ligne, on va vous reprendre, on a compris l'essentiel de votre propos

39. monsieur Janier on va essayer d'établir une meilleure ligne Jean-Christophe Ruffin quelques Jean

40. Christophe Ruffin quelle a été votre expérience vous ?

\footnotetext{
${ }^{9} \mathrm{~T}$ correspond à Thomas, auditeur qui intervient sur l'antenne, PB - Patrick Boyer, animateur, AJ - André Janier, JCR - Jean-Christophe Rufin, FR - Franck Renaud.
} 
41. JCR : Moi je pense qu'au contraire en Afrique bon indépendamment de la question économique parce

42. que bien sûr quand il s'agit de promouvoir ses entreprises chacun a une préférence pour ses

43. entreprises nationales ce qui est normal mais mais sur le plan politique je je pense que notamment en

44. Afrique notamment dans les anciennes colonies françaises il est très très important $\mathrm{e}$ d'agir de façon

45. coordonnée avec les autres pays européens parce que je prends un exemple si vous voulez faire des

46. commentaires sur des affaires de corruption par exemple dans le pays ou sur des points sensibles de la

47. vie politique d'un pays moi j'étais au Sénégal dans un pays où se profile une succession e difficile

48. PB : Dynastique osons le mot

49. JCR : Qui peut-être (rire) serait dynastique lorsque vous voulez intervenir sur ce sujet si vous êtes

50. l'ambassadeur de France e vous êtes l'ancien colonisateur et immédiatement vous déclenchez des

51. contre-feux terribles on vous dit c'est le colon et cetera qui parle et cetera bon si vous avez une

52. démarche européenne vous avez une démarche concertée vous pouvez faire passer des messages

53. beaucoup plus facilement je l'ai personnellement expérimenté je crois qu' on peut être beaucoup plus

54. convaincant et de cette manière beaucoup plus acceptable même dans ces remarques si on est si on

55. est si on joue le jeu européen à fond

La construction conjointe du sens par les interlocuteurs se manifeste d'abord par le maintien du même thème effectué au moyen de la reprise de la notion de coopération, de sa définition et redéfinitions successives ainsi que par sa contextualisation. Ainsi, les expressions employées par l'auditeur dans les lignes 7 et 8 la collaboration et la synergie sont reformulées avec des formulations plus simples ils travaillent ensemble par l'animateur dans la ligne 10. Le premier intervenant sollicite ${ }^{10}$ restreint dans la ligne 10-11 la coopération entre les ambassadeurs à des réunions sur des sujets techniques et au contexte du Vietnam qu'il connaît, mais en même temps il introduit dans les lignes 14-15 un aspect nouveau et, comme on pourra s'en rendre compte dans la suite de l'échange, pertinent et éclairant pour le sujet, à savoir celui du poids et de l'impact de certains pays par rapport aux autres ; ici il s'agit de la présidence des réunions de collaboration: c'est la France ou l'autre pays c'est l'ambassade du pays qui préside l'Union européenne qui accueille ces réunions. L'idée de synergie technique est constituée en objet de discours par l'animateur et opposée dans les lignes 16-17 à une complicité une solidarité même des pays européens. L'interlocuteur interpelé évalue dans la ligne 18 positivement cette proposition et encore une fois la situe dans le contexte de son expérience de travail diplomatique à Téhéran ${ }^{11}$.

\footnotetext{
${ }^{10}$ Notons au passage la quasi absence de la négociation des tours qui sont distribués, conformément au genre de l'émission, par l'animateur.

${ }^{11}$ Il est intéressant de noter la manière, par excellence diplomatique, de qualifier le régime autoritaire d'Iran de situation un peu spéciale et d'appeler raisons historiques la rupture des relations diplomatiques entre les États-Unis et l'Iran, ces expressions loin de dire les choses explicitement font référence aux représentations supposées partagées par les auditeurs. Dans la suite de l'émission, la référence au contexte colonial de la France est d'ailleurs aussi en partie implicite et peut, de ce fait, rester opaque pour certains étudiants polonais, la sensibilisation à l'implicite constitue donc un défi de taille pour les apprenants.
} 
La conceptualisation de coopération est poursuivie (lignes 29-37) par AJ qui dénonce une différence entre la coordination, la solidarité affichées et la manière dont les affaires secrètes se traitent sur le terrain. L'ambassadeur AJ ayant l'expérience de l'Afrique et du Proche Orient évalue explicitement les propos de son (ses) prédécesseur(s) ${ }^{12}$. Dans son explication, $\mathrm{AJ}$ réinterprète la coopération, décrite par ses collègues comme apparente et superficielle, voire inexistante là où les intérêts économiques des pays particuliers entrent en jeu (lignes 33-37). Plus loin, à la ligne 39, AJ ira jusqu'à opposer la concurrence exacerbée à la coopération. Dans les lignes 44-45 l'ambassadeur suivant, JCR, ratifie en partie les propos de AJ non pour les appuyer mais probablement pour faire remarquer leur naïveté : bien sûr quand il s'agit de promouvoir ses entreprises chacun a une préférence pour ses entreprises nationales ce qui est normal. En effet, il suggère (encore une fois implicitement) que les attentes de coopération économique n'ont pas vraiment lieu d'exister et que la seule coopération réelle et valable est celle politique. Il appuie ce constat avec un ample exemple d'actions diplomatiques dans les anciennes colonies de la France (lignes 47-57).

Sur le plan didactique, les procédés interactifs analysés ci-dessus peuvent constituer à bien des égards des exemples de l'élaboration commune du sens. En plus, le travail d'interaction concerne dans l'émission proposée les situations de parole partiellement planifiées à l'avance et donc préfabriquées (comme c'est le cas des auditeurs qui appellent) mais qui en même temps doivent être constamment adaptées à l'évolution de la rencontre (les experts qui tout en ayant fait une réflexion préalable sur le sujet doivent aussi réagir aux propos des autres intervenants de manière plus spontanée). L'intérêt didactique de ce type de séquences consiste dans le fait qu'elles montrent comment conjuguer la préparation à la prise de parole (forme et contenu) des apprenants sans pour autant enlever à cette activité son caractère de construction située en interaction avec autrui.

\subsection{L'ACCÈS AUX REPRÉSENTATIONS - ENTRE OBJECTIVITÉ ET SUBJECTIVITÉ}

L'interaction sur le contenu (thème, opinion) suppose une double interactivité (Vion, 1992) : l'auditeur non seulement doit suivre la construction conjointe du contenu par les interlocuteurs dans l'émission, mais il doit aussi l'analyser et l'évaluer en fonction de ses propres représentations de la problématique et en faisant appel à ses compétences médiatiques pour bien saisir les enjeux de l'émission et déjouer d'éventuelles tentatives de manipulation.

\footnotetext{
${ }^{12}$ Il le fait par ailleurs en le précédant de procédés de figuration dans les lignes 27 et 29 consistant à marquer son hésitation et finalement répondant à l'encouragement de l'animateur dans la ligne 28.
} 
Pour gagner l'adhésion des auditeurs aux thèses présentées dans l'émission, les journalistes et les invités doivent tenir compte des valeurs et des convictions sociales du public tel qu'ils l'imaginent. Les activités d'analyse de la problématique exposée dans le chapeau devraient préparer les apprenants/auditeurs à saisir l'orientation argumentative prévue par les instances de production et à découvrir les représentations sous-jacentes. La manière de réagir des invités à la description de la problématique peut être révélatrice de ces représentations partagées. Pour illustrer cet aspect, considérons le chapeau d'Alain Bedouet dans l'introduction de l'émission consacrée au chômage des jeunes ${ }^{13}$ du mardi 31 août 2010 (0 $\min 36$ de $\left.2 \min 20\right)$ :

1. Bonsoir c'est un message de Karine à Nancy dont j'extrais l'essentiel mais qui commence ainsi à lire tant et

2. tant d'articles sur les Roms on avait fini par croire que les vrais problèmes ceux de l'emploi et du pouvoir

3. d'achat étaient enfin réglés et pourtant le chômage des jeunes devient dramatique y compris ceux qui ont

4. des diplômes pourtant reconnus et recherchés c'est le cas de mon fils 25 ans qui vit à la maison et est depuis

5. 20 mois maintenant à la recherche d'emploi ma fille 22 ans bac plus 5 galère elle aussi de petits boulots de

6. remplacements en attendant un vrai travail et Karine ajoute ils sont patients ces jeunes mais nous les parents

7. sommes nerveusement épuisés complètement angoissés et d'ailleurs nous ployons sous la charge car tout

8. juste retraitée je dois à la fois aider ces deux enfants mais aussi payer la maison de retraite de mon père

9. C'est donc au chômage des jeunes 18-25 ans sont-ils devenus ou en train de devenir une sorte de génération

10. sacrifiée car le manque de travail la précarité de l'emploi font des ravages le chômage de longue durée

11. accuse une hausse parmi les moins de 25 et d'ailleurs il faut le dire pas seulement en France, le BIT le

12. bureau international du travail a noté que sur 620 millions des jeunes économiquement actifs dans le

13. monde 81 millions sont sans emploi un niveau encore jamais enregistré

Nous avons affaire ici à une introduction doublement subjective qui correspond à l'enjeu de captation plutôt qu'à l'enjeu d'information (Charaudeau, 2006). D'abord, on a choisi parmi les e-mails envoyés par les auditeurs celui qui présente une situation difficile - une mère dont les deux enfants, malgré leurs diplômes, sont au chômage. Dans la lettre citée, l'auteure généralise sa situation particulière dans les énoncés suivants : le chômage des jeunes devient dramatique à la ligne 3 , nous les parents nous sommes nerveusement épuisés complètement angoissés et d'ailleurs nous ployons sous la charge à la ligne 7. Après avoir lu la lettre, l'animateur Alain Bédouet pose la problématique pratiquement dans le même style que celui de la citation, empreint de modalisations fortes et fréquentes pour ne citer que les lignes 9-10 une sorte de génération sacrifiée, à la ligne 10 le manque de travail la préca-

\footnotetext{
${ }^{13}$ Le titre de cette émission est Chômage des jeunes : les 18-25 ans sont-ils devenus une "génération sacrifiée »? Ont participé à cette émission les invités suivants : Anne Muxel, directrice de recherche au Cnrs-Cevipof, Eric Heyer, directeur adjoint de l'OFCE, Observatoire français des conjonctures économiques, Ophélie Natil, membre du Collectif « Génération précaire », ainsi que Anna Melin, secrétaire générale de l'Unef, Marie-Hélène Ballestero, correspondante de France Inter à Madrid et Angélique Kourounis, correspondante à Athènes.
} 
rité de l'emploi font des ravages. Le tout se termine par des chiffres qui concernent le monde entier et dont on ne sait pas déduire ceux qui concernent la France.

Nous n'avons pas de place ici pour analyser l'ensemble de l'émission mais l'interprétation proposée par le journaliste domine largement la réflexion développée par les invités dans le sens de la déresponsabilisation des jeunes impuissants par rapport à cette situation. Les quelques voix qui tentent de dédramatiser la situation et de montrer le revers de la médaille sont pratiquement étouffées. La réduction de la rencontre à des considérations qui visaient l'État comme le seul responsable de la situation des jeunes, vus tous comme un groupe tout aussi homogène qu'impuissant, nous fait penser qu'il peut s'agir ici des représentations tellement partagées par un grand groupe de personnes (les auditeurs de France Inter? les Français ?) qu'elles ne font plus objet de questionnement. Pour un auditeur/apprenant polonais cela pourrait donner lieu à une hypothèse sur une vision culturellement différente du rôle de l'État et de la responsabilité individuelle, engendrant des attentes envers les institutions, hypothèse à vérifier, bien évidemment.

\section{LA DIDACTISATION DES PODCASTS}

Les interactions comme lieu d'acquisition langagière et d'apprentissage ne constituent pas une idée nouvelle dans le champ de la didactique. La majorité des études portent pourtant sur l'acquisition des éléments du système linguistique (Séquences Potentiellement Acquisitionnelles - De Pietro et al., 1989), en faisant abstraction des compétences de haut niveau de gestion interactive des contenus, perspective qui nous intéresse ici. Le travail sur les modes de construction du sens social présente l'avantage de sortir des sentiers battus des activités proposées aux étudiants avancés, trop souvent mis en situation de restitution des connaissances, conçues comme statiques et objectives, sans tenir compte du caractère construit de ces connaissances et des limites de celles-ci. Par cet aspect, le travail à partir des podcasts de radio apparaît comme une alternative fort valable au modèle du développement de l'oral, le plus fréquent actuellement, où la préparation à la prise de parole se fait presque exclusivement à partir des textes écrits (articles de presse, billets de blog, etc.) à caractère monologal. Les tâches proposées dans ce modèle sont trop souvent basées soit (1) sur une préplanification définitive de la parole, soit (2) sur des prises de parole complètement spontanées avec emploi de procédés interactifs élaborés dans la langue maternelle, souvent intuitifs et insuffisants dans le contexte interculturel. Sans parler du fait que l'attention de l'apprenant est focalisée sur l'acquisition de la richesse lexicale et syntaxique qui, pour importante qu'elle soit, n'épuise pas à elle seule la compétence de communication orale.

Dans l'approche proposée ici, en revanche, le développement des compétences orales se ferait à travers la bifocalisation sur le sens et les procédés interactifs de sa 
construction. Le modèle proposé reflète donc, partiellement, les idées exposées par W. Wilczyńska (2002). Il aurait pour objectifs de mettre progressivement en place des capacités telles que :

- la saisie et la compréhension de l'intention des locuteurs, l'identification des thèmes principaux et de la valeur pragmatique de leur sélection et de leur utilisation en fonction d'une intention donnée; la détection de toutes sortes de manipulations ou de tentatives d'influence,

- le développement des procédés de négociation des contenus mis en circulation par les interlocuteurs dans le respect des normes (y compris au niveau interpersonnel et organisationnel) du genre discursif donné,

- l'enrichissement de représentations du monde propres aux étudiants (souvent restant sous l'impact de la L1 et de sa culture) dans un mouvement de confrontation aux contenus médiatiques francophones.

Sur le plan cognitif, ce qui n'est pas à sous-estimer, les procédés de contrôle du sens et de sa négociation présentent des ressemblances notables, en améliorant l'économie d'enseignement/apprentissage. Bien évidemment, ces procédés exigeront des formes langagières (ainsi que des formes de figuration et d'organisation) diverses en fonction des genres et de leurs variantes réalisées ${ }^{14}$. Les procédés de coconstruction du sens en interaction demanderont donc à chaque fois un réancrage contextuel, comme c'est d'ailleurs le cas pour toute capacité de communication qui, pour reprendre la formulation de Mondanda et Pekarek-Doehler, est située, changeante selon les contextes et donc ne fonctionne pas selon une logique du transfert d'un contexte à un autre mais plutôt selon une logique de réancrage, appropriation située, d'exploitation indexale (Mondada, Pekarek-Doehler, 2001, p. 117).

C'est pour cette raison qu'il nous paraît judicieux de prévoir l'écoute et l'analyse des procédés interactifs dans différentes émissions du même type pour permettre à l'apprenant d'observer ce qui est stable, répétitif (et donc propre au genre médiatique en question) et ce qui est sujet à variation sous l'impact de la configuration des interlocuteurs (rôles, rapports: p. ex. interlocuteurs intervenant en tant que représentants d'une institution ou à titre personnel), de l'objet du discours et de l'orientation dominante (information vs. influence). L'analyse de l'émission Le téléphone sonne dont nous avons présenté un modeste échantillon dans cet article, nous confirme dans le choix des podcasts de radio d'information et d'opinion comme source de modèles de discours à proposer aux apprenants adultes au niveau avancé (C1).

\footnotetext{
${ }^{14}$ Ainsi, on pourra trouver un procédé d'évaluation des propos d'autrui exprimés aussi bien dans le registre familier que dans le registre soutenu, soigneux de ménager la face (discussion) ou au contraire fort menaçant pour la face de l'interlocuteur (dispute, injure).
} 


\section{BIBLIOGRAPHIE}

Bange, P. (1992). Analyse conversationnelle et théorie de l'action. Paris : Hatier-Didier.

Becqueret, N. (2002). Les émissions interactives à la radio : la parole par téléphone. Quelle parole ? Les cahiers du CREDAM, 2, 85-91.

Becqueret, N. (2004). La parole des auditeurs dans les émissions de radio informatives en France : Entre tradition républicaine et tentation libérale. In Questionner l'internationalisation - Actes du XIV Congrès national des sciences de l'information et de la communication (pp. 103-110). Béziers.

Bronckart, J.-P. (1996a). Activité langagière, textes et discours. Pour un interactionnisme sociodiscursif. Paris : Delchaux et Niestlé.

Bronckart, J.-P. (1996b). L'acquisition des discours. Le point de vue de l'interactionnisme sociodiscursif. Le Français dans le Monde. Recherches et Applications « Le discours : enjeux et perspectives », 55-64.

Burger, M. (2010). Le cadrage de la communication dans les médias. Communication, 27/2, 18-50.

Burger, M. (2013). Vers une analyse de la construction des identités dans les discours de communication publique. Cahiers de l'ILSL, 34, 101-122.

Charaudeau, P. (1997). Le discours d'information médiatique : la construction du miroir social. Paris : Nathan / Institut national de l'audiovisuel (coll. « Médias-Recherches »).

Charaudeau, P. (2006). Discours journalistique et positionnements énonciatifs. Frontières et dérives. SEMEN, 22.

Charaudeau, P. (2011). Les médias et l'information. Bruxelles : De Boeck.

De Pietro, J-F., Matthey, M., Py, B. (1989). Acquisition et contrat didactique : les séquences potentiellement acquisitionnelles dans la conversation exolingue. In Actes du 3e Colloque Régional de Linguistique (pp. 99-124). Strasbourg : Université des Sciences Humaines et Université Pasteur.

Górecka, J. (2006). Développer la pensée critique à travers les discussions en langue étrangère. Kraków : Flair.

Górecka, J. (2008). Podcast jako przykład wykorzystania nowych technologii w dydaktyce języków obcych: motywowanie uczących się poprzez tworzenie spójnego i otwartego środowiska uczenia się. In A. Michońska-Stadnik, Z. Wąsik (réd.), Nowe spojrzenia na motywację $w$ dydaktyce języków obcych. T. 2.2 (pp. 87-96). Wrocław : Wydawnictwo Wyższej Szkoły Filologicznej we Wrocławiu.

Kerbrat-Orecchioni, C. (2005). Discours en interaction. Paris : Armand Colin.

Maingueneau, D. (2014). Discours et analyse du discours. Paris : Armand Colin.

Mondada, L., Pekarek-Doehler, S. (2001). Interactions acquisitionnelles en contexte: perspectives théoriques et enjeux didactiques. Le Français dans le Monde, $n^{o}$ spécial, Théories linguistique et enseignement du frnaçais aux non francofomes, 107-142.

Parlement européen et Conseil (2006). Recommandation du Parlement Européen et du Conseil du 18 décembre 2006 sur les compétences clés pour l'éducation et la formation tout au long de la vie (/962/CE). URL : http://eur-lex.europa.eu/LexUriServ/LexUriServ.do?uri=OJ:L:2006:394:0010: 0018:fr:PDF

Pekarek, S. (1999). Leçons de conversations. Dynamiques de l'interaction et acquisition de compétences discursives en classe de langue. Fribourg : Éditions Universitaires de Fribourg.

Pekarek-Doehler, S. (2002). Formes d'interaction et complexité des tâches discursives : les activités des tâches conversationnelles en classe de langue. In F. Cicurel (réd.). Discours, action et appropriation des langues (pp. 117-130). Paris : Presses Sorbonne Nouvelle.

Py, B. (1990). Les stratégies d'acquisition en situation d'interaction. Le Français dans le monde, $n^{\circ}$ spécial, 81-88. 
Py, B., Jeanneret, T. (1999). Apprentissage de la syntaxe dans l'interaction exolingue. In Communication au XI Colloque international: Usages pragmatiques et acquisition des langues étrangères (pp. 19-21). Paris.

Vasseur, M.-T. (1993). Gestion de l'interaction, activités métalangagières et apprentissage en langue étrangère. Acquisition et interaction en langue étrangère, 2, 25-59.

Vion, R. (1992). La Communication verbale. Analyse des interactions. Paris : Hachette Supérieur.

Wilczyńska, W. (2002). Autonomizacja $w$ dydaktyce języków obcych. Doskonalenie się w komunikacji ustnej. Poznań : Wydawnictwo Naukowe UAM.

Wojciechowska, B. (2007). Le développement de la sensibilité langagière en communication orale exolingue au niveau avancé. Thèse de doctorat non publiée. 